\title{
Purification of age-distinct insulin secretory granules through antigen restriction using the CLIP-tag
}

Martin Neukam ${ }^{1,2,3}$, Katharina Gan $\aleph^{1,2,3}$, Jovana Vasiljević ${ }^{1,2,3}$, Johannes Broichhagen ${ }^{4,5}$, Kai Johnsson ${ }^{4}$, Thomas Kurth ${ }^{6}$ and Michele Solimena ${ }^{1,2,3,7 \#}$

${ }^{1}$ Molecular Diabetology, University Hospital and Faculty of Medicine Carl Gustav Carus, TU Dresden, Dresden, Germany

${ }^{2}$ Paul Langerhans Institute Dresden (PLID) of the Helmholtz Center Munich at the University Hospital Carl Gustav Carus and Faculty of Medicine of the TU Dresden, Dresden, Germany ${ }^{3}$ German Center for Diabetes Research (DZD e.V.), Neuherberg, Germany

${ }^{4}$ Max-Planck-Institute for Medical Research, Department of Chemical Biology, Heidelberg, Germany

${ }^{5}$ Present address: Forschungsinstitut für Molekulare Pharmakologie, Department of Chemical Biology, Berlin, Germany.

${ }^{6} \mathrm{TU}$ Dresden, Center for Molecular and Cellular Bioengineering (CMCB), Technology Platform, Dresden, Germany

${ }^{7}$ Max Planck Institute for Molecular Cell Biology and Genetics, Dresden, Germany

\#Corresponding author.

Tel.: +49-351-796-366-11

e-mail: Michele.Solimena@uniklinikum-dresden.de 


\begin{abstract}
Pancreatic islet beta cells employ secretory granules for the storage and glucose-stimulated release of the hormone insulin. The competence of an insulin granule for exocytosis depends on spatial and temporal variables such as its proximity to the plasma membrane as well as its age, with newly-generated granules being preferentially released. The molecular underpinnings for the control of these variables remain largely unknown and their uncovering is of high relevance for the study of diabetes, which results from deficient insulin secretion. However, we still lack a comprehensive view about the molecular composition of the insulin granules and how this may change over their lifetime. Here we report a strategy for the background-free purification of insulin secretory granules of distinct age from insulinoma INS1 cells. We show that utilization of an immuno-based affinity approach for pulse-chase labeled insulin secretory granules, produces a highly enriched granular fraction. Our approach precludes typical contaminants from the solid phase and may be designed to purify secretory granules of a distinct age.
\end{abstract}




\section{Introduction}

Insulin is the main hormone for induction of glucose uptake into muscle cells and adipocytes. Therefore, its glucose-stimulated secretion from pancreatic islet beta cells is critical for maintenance of blood glucose homeostasis. Insulin is produced as a prohormone precursor in the endoplasmic reticulum (ER) of beta cells and delivered via the Golgi complex to the immature insulin secretory granules (SGs). Activation of prohormone convertases upon acidification of the immature SG lumen drives the conversion of proinsulin into insulin, and thereby SG maturation. Mature insulin SGs are then stored in the cytoplasm until hyperglycemia triggers their exocytosis. Insulin SGs differ according to their competence for exocytosis and can be divided into several distinct functional pools, mainly based on their location. SGs close to the plasma membrane, in particular, are considered more likely to undergo exocytosis ${ }^{1,2}$. Recently, however, there has been renewed interest in the notion of newly-synthesized SGs having greater propensity for exocytosis than their older counterparts ${ }^{3,4,5}$. Specifically, we established that younger SGs are more mobile and acidic relative to aged $S G s^{6,7}$ - two properties which might contribute to their preferential release. However, the reasons why older SGs are less prone to be recruited for exocytosis, and are instead increasingly removed by autophagy ${ }^{8}$, remain unknown. Therefore, the identification of molecular signatures for SG aging is a major outstanding question in the field of insulin secretion, and peptide-hormone/neuropeptide secretion in general. Progress in this area has however been hampered by the lack of protocols for the purification of SGs of defined age for subsequent proteomic and lipidomic analyses.

Previous attempts to isolate insulin SGs for proteomic analysis have identified $50-100$ SG proteins, including many established cargoes of these organelles and several new candidate components $^{9,10,11}$. However, many non-SG proteins were also detected, while several wellknown SG cargoes were not detected ${ }^{12}$. Because of their technical limitations, typical enrichment protocols for organelles are prone to cross-contamination. The most widely applied method for organelle purification is subcellular fractionation, either by differential or gradient centrifugation. Here, physical properties, such as weight, size and buoyancy, are exploited to separate and enrich specific organelles. Both procedures, however, cannot avoid contamination with other cellular compartments that have similar physical properties, especially vesicular organelles, such as lysosomes, synaptic-like microvesicles or endosomes. An alternative approach is the immunoisolation of organelles with antibodies that recognize a specific epitope in the cytoplasmic domain of a transmembrane bait protein, followed by the binding of the antibody to a solid phase $e^{13,14,15}$. Since copies of post-Golgi vesicle transmembrane proteins are also in transit through the ER and the Golgi complex at 
the time of cell lysis, contamination by the latter organelles is nonetheless possible. All of these limitations reduce the accuracy of downstream proteomic and lipidomic analyses.

Here, we report an immuno-based approach for the purification of insulin SGs of distinct age. Our protocol takes advantage of the specificity of immunopurification and combines it with pulse-chase labeling to restrict the antigen to post-Golgi organelles using a CLIP-tag. We show that our approach dramatically reduces background, giving access to pure SG fractions that can be eluted as intact organelles. This approach is suitable for the isolation of pools of age-distinct insulin SGs, which would allow the search for molecular signatures related to their different properties.

\section{Results}

\section{Strategy}

Immunopurification protocols provide high specificity for the respective antigen, but often suffer from contamination by pull-down of pre-SG compartments and unspecific binding to magnetic beads. To address those shortcomings, we fused the CLIP-tag to the cytoplasmic C-terminus of phogrin, an intrinsic membrane protein of the SGs (fig 1A). CLIP is a self-labeling protein which can covalently bind to a cell-permeable CLIP-substrate in a pulse-chase manner, thereby ensuring that only the newly synthesized phogrin-CLIP is labeled. Cells were then incubated until the newly synthesized and labeled phogrin-CLIP exited the Golgi complex and it is sorted into SGs. Varying the time interval between the labeling of newly synthesized phogrin-CLIP and cell lysis it is possible to obtain extracts in which only a time-resolved pool of SGs contains the labeled phogrin-CLIP bait. Using an antibody against the CLIP-substrate, such as the fluorescent dye tetramethylrhodamine (TMR), rather than the bait protein allows for both the purification of age-distinct SGs and the exclusion of contamination from pre-SG compartments. To reduce background by non-specific binding to the magnetic beads, we further included a highly specific protease cleavage site between the CLIP-tag and the cytoplasmic domain of phogrin allowing the restricted elution of the immunoisolated organelles (fig 1C). We chose phogrin as a specific bait of SGs, because, unlike its paralogue islet cell autoantigen 512 (ICA512) $^{16}$, it is not susceptible to proteolytic cleavage by calpain upon its transient insertion to the plasma membrane ${ }^{17}$ (and our unpublished observations), and thereby potentially depleted in older SGs following repeated rounds of kiss-and-run or partial exocytosis. Hence, phogrin is an optimal candidate for the cytoplasmic addition of a CLIP-tag at its C-terminus. Since phogrin has been proposed to have phosphatidylinositol phosphatase activity $^{18}$, we replaced the catalytic cysteine 931 with a serine ${ }^{18}$ to avoid interferences with potential lipidomic analyses by overexpression of our reporter. Apart from being able to 
distinguish SGs based on their age, the CLIP tag simultaneously allows for the specific labeling of post-Golgi compartments. This principle appears to be key for the dramatic reduction of non-SG organelles being co-purified.

\section{Microscopic and biochemical characterization of phogrin-CLIP}

First, we tested the subcellular localization of phogrin-CLIP C931S (henceforth termed phogrin-CLIP) to ensure its efficient and specific targeting to insulin SGs. Structured illumination microscopy (SIM) revealed that phogrin-CLIP covalently labeled with the dye BCTMR was found in punctate structures which almost exclusively co-localized with endogenous insulin (fig 1B-C, S1A-C). We did not detect significant co-localization with early endosomal and lysosomal markers EEA1 and LAMP2, respectively (fig 1C, S1B-C). Co-labeling for CLIP with an antibody served as a positive control (fig 1C, S1A) and revealed that not all fusion proteins were positive for TMR, i.e. that washing of the substrate was sufficient to prevent continuous labeling (fig S1A).

Next, we analyzed some of the properties of a stable phogrin-CLIP INS-1 cell line. As expected, we could only detect the CLIP signal in the stable cell line and not in non-transfected control samples (fig S2C). Addition of the $20 \mathrm{kDa}$ CLIP tag retarded the electrophoretic mobility of pro-phogrin by SDS-PAGE to an apparent molecular weight of $\sim 120 \mathrm{kDa}$ and that of mature phogrin to $\sim 80 \mathrm{kDa}$ (fig $\mathrm{S} 2 \mathrm{C}$ ). Probing for untagged phogrin, we found that the stably expressing phogrin-CLIP INS-1 cells retained the expression of endogenous phogrin similar to control cells, as shown by quantitative PCR and immunoblot (fig S2B-C). Since phogrinCLIP was more abundant than endogenous phogrin, we tested whether its overexpression affected the levels of other insulin SG components and insulin secretion. Compared to nontransfected INS-1 cells, phogrin-CLIP INS-1 cells did not display obvious changes in the content of chromogranin A (ChgA), prohormone convertase 2 (PC2) or carboxypeptidase $E$ (CPE), as shown by immunoblot (fig S2D). However, stable overexpression of phogrin-CLIP correlated with increased glucose-stimulated insulin secretion (GSIS), whereas insulin biosynthesis was unaffected (GSTI, fig S2E).

We further assessed the glycosylation status of phogrin-CLIP as an independent measure of its trafficking and conversion. Proteins in the secretory pathway are N-glycosylated, a posttranslational modification which is later modified along the secretory pathway. Different glycosidases may or may not be able to cleave these sugar complexes based on their composition. The peptide:N-glycosidase F (PNGaseF) cleaves all N-glycosylations independent of their composition, whereas the Endoglycosidase $\mathrm{H}(\mathrm{EndoH})$ only cleaves glycosylations that are not modified by the Golgi-resident alpha-mannosidase II i.e. 
glycosylated secretory proteins on their way from the ER to the Golgi complex. The glycosylation status can therefore be used to determine if a protein has been transported at least to the Golgi. Rat and mouse phogrin are predicted to be N-glycosylated at N553 and N550, respectively. Upon digestion with PNGaseF, both pro-phogrin-CLIP and phogrin-CLIP displayed a faster electrophoretic mobility, consistent with being $\mathrm{N}$-glycosylated. Mature phogrin-CLIP, but not pro-phogrin-CLIP, was instead insensitive to EndoH treatment (fig S2A), indicating, together with conversion, its progression and maturation along the secretory pathway.

Finally, we transfected the stable phogrin-CLIP line with our previously described insulin SG age-reporter human insulin-SNAP ${ }^{5}$ (Ins-SNAP) to test whether the two reporters would have similar localization and SG aging kinetics. When CLIP and SNAP tags were simultaneously labeled with orthogonal dyes BC-TMR and SNAP-Cell ${ }^{\circledR} 430$, respectively, for an SG age of 2$4 \mathrm{~h}$ we could detect a high level of correlation (fig S3A, C). However, when labeling Ins-SNAP for 20-22 $\mathrm{h}$ of age and phogrin-CLIP for an age of 2-4 $\mathrm{h}$ we could not detect any significant correlation and the signals were clearly distinct (fig S3B-C).

Taken together, we conclude that phogrin-CLIP is transferred efficiently to the Golgi and postGolgi compartments and that its stable overexpression does not significantly affect insulin SG stores or secretion. It also has similar kinetics to Ins-SNAP and can therefore serve as reliable bait for the immunoisolation of age-distinct insulin SGs.

\section{Immunopurification of phogrin-CLIP and SGs}

Despite the use of specific antibodies, immunoisolation protocols often suffer from non-specific co-enrichment. This is due to non-specific binding of the antibodies, as well as unintended binding of proteins to the solid phase, such as magnetic beads, during the isolation procedure. To reduce the latter, we incorporated a site-specific protease cleavage site into phogrin-CLIP (fig 1A), which allows for the separation of SGs from the beads. Using silver staining after SDS-PAGE, we assessed the degree of background of immunoisolated labeled and nonlabeled samples. As expected, the input and flow-through in both TMR positive and negative samples appeared similar (fig 2A) and showed a plethora of bands. Similarly, beads from both samples showed distinct protein bands, which, however, were slightly more abundant in the TMR positive samples. The eluates, when released from the beads by the protease, were instead remarkably different. Apart from a distinct band around $25 \mathrm{kDa}$, representing the added protease, no protein band was visible in the eluate of the beads incubated with the unlabeled phogrin-CLIP control sample. In contrast, the eluate of the beads incubated with the TMR labeled phogrin-CLIP sample showed a multitude of bands (fig 2A). 
To verify the selective immunoisolation and elution of SGs, we immunoblotted control and labeled fractions for markers of distinct intracellular compartments. The eluate from the TMR labeled phogrin-CLIP sample, but not that from the control sample, was enriched for the presence of several granule cargoes, including CPE, PC2, ChgA, ICA512 and phogrin (fig 2BD, fig S4A-C, fig S5A). It was negative instead for Golgi markers TGN38 and GM130, ER markers CANX and PDI (fig 2C, D, fig S4A, C), as well as the early endosome marker EEA1 (fig 2C). Interestingly, we could also detect the immature SG marker islet cell autoantigen $69^{19,20}$ (ICA69, fig S4A). As expected from the silver staining, none of the mentioned markers were detected in the eluate from the control beads. We could, however, detect the lysosomal marker LAMP2 and the synaptic-like microvesicle marker synaptophysin 1 (Syp1, fig S5A). These markers may arise from degradation of labeled SGs in lysosomes (LAMP2) or sorting of phogrin-CLIP into synaptic-like microvesicles (Syp1). To avoid co-purification of these organelles, we included a second immunoisolation step with antibodies targeting the cytoplasmic tails of either LAMP2 alone or a combination of LAMP2 and Syp1. Interestingly, both proteins could be depleted by the immunoisolation with the anti-LAMP2 antibody alone, suggesting that both markers resided in a common organelle, presumably lysosomes (fig $\mathrm{S} 5 \mathrm{~B})$. This data indicates that the only non-SG organelle present in our isolation is a fraction of labeled SGs undergoing degradation in lysosomes, which, however, could be immunodepleted with additional LAMP2 antibodies.

Finally, we evaluated the ultrastructural integrity of the purified SGs. Aliquots of the beads of TMR-labeled samples were embedded in epon and ultrathin sections were imaged by electron microscopy (fig 3A-B), or eluted SGs were spotted on grids for negative staining (fig 3C-D). Electron micrographs confirmed the purity and integrity of the immunoisolated SGs, with no obvious contamination from other organelles. Epon-embedded SGs bound to beads contained the characteristic dense core of insulin granules surrounded by a lipid bilayer (fig 3B). In summary, these data indicate that our protocol allows for the highly specific and backgroundfree enrichment of insulin SGs from INS-1 cells using a cytoplasmic CLIP tag.

\section{Discussion}

The purification of synaptic vesicles to homogeneity ${ }^{21}$ has been a milestone in the field of cell biology and has paved the way to the identification of key factors involved in the assembly and regulation of SNARE complexes such as synaptobrevin/NAMP2 ${ }^{22}$ and synaptotagmin. Since then, there have been multiple attempts to similarly obtain pure preparations of neuronal largedense core vesicles or peptide hormone-containing granules from different endocrine sources, 
such as chromaffin and pheochromocytoma cells, the posterior pituitary or insulin secreting insulinoma cells. Although substantial progress has been made in enriching granules, none of the different strategies has succeeded in producing a highly purified fraction ${ }^{12}$.

Despite very sophisticated combinations of density centrifugation ${ }^{9}$ and additional combination with SILAC ${ }^{11}$, typical contaminants remain an issue. Density centrifugation is a powerful technique for the enrichment of certain organelles, but their distinct separation can be challenging. Organelles can have different physical properties, sizes and densities, as for example organelles of the endo-lysosomal pathway or mitochondria. Consequently, contamination by these organelles is very common and lowers the accuracy of the data obtained. Additional immunopurification ${ }^{10}$ targeting synaptobrevin/VAMP2 was used to select SGs containing the cytoplasmic epitope of VAMP2. However, even in this scenario, VAMP2 is not solely, albeit enriched, restricted to granules, but is part of the secretory pathway and even synaptic-like microvesicles. Most studies were able to report great enrichment efficiency and identified novel granule-associated proteins. However, typical contaminants were always present ${ }^{12}$, which in downstream applications, such as mass spectrometry of proteins and lipids can lead to false-positive attributions, as the quantity of detected proteins/lipids does not necessarily reflect the abundance of a given organelle in the examined material.

Antibodies are highly specific tools to purify organelles regardless of the physical properties of the latter. However, transmembrane proteins travel along the secretory pathway and therefore are not only found on the desired organelle at the time of cell disruption. Thus, the presence of the antigen in compartments such as the ER and the Golgi complex will inevitably result in their co-immunoisolation from the cell homogenates. To avoid these contaminations, we utilized the specificity of phogrin for targeting of the probe to SGs and the attached CLIP tag for purification from insulinoma INS-1 cells. Rather than using antibodies against CLIP, we use its substrate as antigen for affinity purification. With pulse-chase labeling of the CLIP tag by substrates, such as TMR or Fluorescein, only newly-synthesized phogrin-CLIP would be labeled. Giving enough time for the fusion protein to mature and translocate to SGs, the antigen is restricted to post-Golgi organelles. This avoids the co-purification of notorious contaminants, such as the Golgi and ER, which were virtually absent in our purified material. Culturing the cells at glucose levels not eliciting secretion further limited the amount of SGs undergoing exocytosis and therefore the amount of phogrin-CLIP ${ }^{T M R}$ resident at the PM and endosomal compartments ${ }^{23,24}$ at the time of cell harvest. Possible remaining contaminants were SGs undergoing fusion with lysosomes, a process known as crinophagy. These could be immunodepleted with antibodies directed against the cytosolic tail of the specific lysosomal membrane protein LAMP2. As a result, we were able to purify SGs of a high degree of purity. 
In addition, our approach allows for the age-specific isolation of SGs, as the time between labeling and harvesting of cells can be varied to a desired age.

Current models of the coordination of granule exocytosis highlight the importance of the spatial distribution of SGs ${ }^{1,2}$. However, it does not take into account that beta cells, amongst other cell types, preferentially release newly-synthesized cargoes such as peptide hormones ${ }^{25,26}$ or neurotransmitters ${ }^{27,28,29,30}$. This indicates that not only a spatial, but also a temporal component is important for coordinated secretion. Although we could previously show that young SGs are more motile ${ }^{6}$ and acidic $^{7}$ than older SGs and that the latter are more likely to be degraded ${ }^{8}$, we lack a deeper understanding of the factors influencing it.

The identification of proteins involved in the aging of SGs, i.e. in their reduced propensity for microtubule-mediated transport and exocytosis ${ }^{5,6}$, may become a tedious procedure if the influence of each of these proteins in the respective processes has to be tested one by one. To this aim, a global approach, such as proteomic profiling of insulin SG fractions of distinct age by mass spectrometry may prove more effective. The need of mass spectrometry is even more critical to assess if and how time alters the lipid composition of SG membranes. Our pulse-chase labeling protocol for the immunoisolation of SGs of desired age will therefore greatly facilitate the discovery of proteins and lipids accounting for the different properties observed between newly-synthesized and aged SGs. As phogrin is widely expressed in peptide hormone and neuropeptide secreting cells, our protocol could be further applicable to other cell types and lines, hence helping to understand better the mechanisms of their regulated secretion. 


\section{References}

1. Rorsman, P. \& Renström, E. Insulin granule dynamics in pancreatic beta cells. Diabetologia 46, 1029-45 (2003).

2. Shibasaki, T. et al. Essential role of Epac2/Rap1 signaling in regulation of insulin granule dynamics by cAMP. Proc Natl Acad Sci U S A 104, 19333-8 (2007).

3. Schatz, H., Nierle, C. \& Pfeiffer, E.F. (Pro-) insulin biosynthesis and release of newly synthesized (pro-) insulin from isolated islets of rat pancreas in the presence of amino acids and sulphonylureas. Eur J Clin Invest 21, 477-85 (1975).

4. Gold, G., Gishizky, M.L. \& Grodsky, G.M. Evidence that glucose "marks" beta cells resulting in preferential release of newly synthesized insulin. Science $218,56-8$ (1982).

5. Ivanova, A. et al. Age-dependent labeling and imaging of insulin secretory granules. Diabetes 62, 3687-96 (2013).

6. Hoboth, P. et al. Aged insulin granules display reduced microtubule-dependent mobility and are disposed within actin-positive multigranular bodies. Proc. Natl. Acad. Sci. 17, 667-76 (2015).

7. Neukam, M. et al. FLIM-based $\mathrm{pH}$ measurements reveal incretin-induced rejuvenation of aged insulin secretory granules. bioRxiv 174391; doi: https://doi.org/10.1101/174391 (2017).

8. Müller, A. et al. A global approach for quantitative super resolution and electron microscopy on cryo and epoxy sections using self-labeling protein tags. Sci Rep 7 , doi: 10.1038/s41598-017-00033-x (2017).

9. Brunner, Y. et al. Proteomics analysis of insulin secretory granules. Mol Cell Proteomics 6, 1007-17 (2007).

10. Hickey, A.J. et al. Proteins associated with immunopurified granules from a model pancreatic islet beta-cell system: proteomic snapshot of an endocrine secretory granule. J Proteome Res 8, 178-86 (2009).

11. Schvartz, D. et al. Improved characterization of the insulin secretory granule proteomes. J Proteomics 75, 4620-31 (2012).

12. Suckale, J. \& Solimena, M. The insulin secretory granule as a signaling hub. Trends Endocrinol Metab 21, 599-609 (2010).

13. Thomas-Reetz, A. et al. A gamma-aminobutyric acid transporter driven by a proton pump is present in synaptic-like microvesicles of pancreatic beta cells. Proc Natl Acad Sci U S A 90 (11), 5317-21 (1993). 
14. Walch-Solimena, C. et al. Synaptotagmin: a membrane constituent of neuropeptide-containing large dense-core vesicles. J Neurosci 13 (9), 3895-903 (1993).

15. Klemm, R.W. et al. Segregation of sphingolipids and sterols during formation of secretory vesicles at the trans-Golgi network. J Cell Biol 185 (4), 601-12 (2009).

16. Ort, T. et al. Dephosphorylation of beta2-syntrophin and $\mathrm{Ca} 2+/$ mu-calpainmediated cleavage of ICA512 upon stimulation of insulin secretion. EMBO J 20 (15), 4013-23 (2001).

17. Taraska, J.W. et al. Secretory granules are recaptured largely intact after stimulated exocytosis in cultured endocrine cells. Proc Natl Acad Sci U S A 100 (4), 2070-5 (2003).

18. Caromile, L.A. et al. The neurosecretory vesicle protein phogrin functions as a phosphatidylinositol phosphatase to regulate insulin secretion. J Biol Chem 285, 10487-96 (2010).

19. Spitzenberger, F. et al. Islet cell autoantigen of $69 \mathrm{kDa}$ is an arfaptin-related protein associated with the Golgi complex of insulinoma INS-1 cells. J Biol Chem 278 (28), 26166-73 (2003).

20. Buffa, L. et al. ICA69 is a novel Rab2 effector regulating ER-Golgi trafficking in insulinoma cells. Eur J Cell Biol 87 (4), 197-209 (2008).

21. Huttner, W.B. et al. Synapsin I (protein I), a nerve terminal-specific phosphoprotein. III. Its association with synaptic vesicles studied in a highly purified synaptic vesicle preparation. J Cell Biol 96, 1374-88 (1983).

22. Baumert, $M$ et al. Synaptobrevin: an integral membrane protein of 18,000 daltons present in small synaptic vesicles of rat brain. EMBO J 8, 379-84 (1989).

23. Vo, Y.P., Hutton, J.C. \& Angleson, J.K. Recycling of the dense-core vesicle membrane protein phogrin in Min6 beta-cells. Biochem Biophys Res Commun 324, 1004-10 (2004).

24. Wasmeier, $C$ et al. An extended tyrosine-targeting motif for endocytosis and recycling of the dense-core vesicle membrane protein phogrin. Traffic $6,474-87$ (2005).

25. MacGregor, R.R., Hamilton, J.W. \& Cohn, D.V. The by-pass of tissue hormone stores during the secretion of newly synthesized parathyroid hormone. Endocrinology 97, 178-88 (1975).

26. Piercy, M. \& Shin, S.H. Newly synthesized prolactin is preferentially secreted by the adenohypophysis in a primary cell culture system. Mol Cell Endocrinol 21, 7584 (1981). 
27. Kopin, I.J. et al. Selective release of newly synthesized norepinephrine from the cat spleen during sympathetic nerve stimulation. J Pharmacol Exp Ther 161, 2718 (1968).

28. Collier, B. The preferential release of newly synthesized transmitter by a sympathetic ganglion. J Physiol 205, 341-52 (1969).

29. Besson, M.J. et al. Release of newly synthesized dopamine from dopaminecontaining terminals in the stratium of the rat. Proc Natl Acad Sci U S A 62, 741-8 (1969).

30. Molenaar, P.C., Nickolson, V.J. \& Polak, R.L. Preferential release of newly synthesized $3 \mathrm{H}$-acetylcholine from rat cerebral cortex slices in vitro. $\mathrm{Br} \mathrm{J}$ Pharmacol 47, 97-108 (1973).

31. Hanker, J.S. et al. Staining tissue for light and electron microscopy by bridging metals with multidentate ligands. Science 152, 1631-1634 (1966).

32. Venable, J.H. \& Coggeshall, R. A simplified lead citrate stain for use in electron microscopy. J Cell Biol 25, 407-408 (1965) 


\section{Material and methods}

\section{Cell culture}

The phogrin-CLIP line was cultured in standard INS-1 medium as described before ${ }^{5,6,7}$.

\section{Cloning of phogrin-CLIP}

Mouse phogrin was obtained from the IMAGE consortium (clone BC_133678) and CLIPf from NEB. The fusion protein was generated by standard molecular cloning techniques. Phogrin was mutated with a site-directed mutagenesis kit (Agilent) to change cysteine 931 to serine as described earlier to abolish phosphatase activity ${ }^{18}$. We then connected phogrin and CLIPf with a PreScission protease cleavage site (LEVLFQGP) flanked by linkers on each side.

\section{Generation of BC-Fluorescein}

A $20 \mathrm{~mL}$ dram vial was charged with $50.0 \mathrm{mg}$ (133 $\mu \mathrm{mol}, 1.0$ equiv.) of isomerically pure 6carboxylfluorescein dissolved in DMSO $(2.5 \mathrm{ml})$ and $87.8 \mu \mathrm{l}(531 \mu \mathrm{mol}, 4.0$ equiv. $)$ of DIPEA before $48.0 \mathrm{mg}$ ( $159 \mu \mathrm{mol}, 1.1$ equiv.) of TSTU was added in one portion. The reaction mixture was allowed to stir for $15 \mathrm{~min}$, before $45.9 \mathrm{mg}$ (199 $\mu \mathrm{mol}, 1.5$ equiv.) of $\mathrm{BC}-\mathrm{NH}_{2}$ was added and stirring was continued for another hour. The reaction was quenched by addition of 5 vol\% HOAc and 10 vol\% water and subjected to RP-HPLC. The product-containing fractions were combined and freeze-dried to obtain $52 \mu \mathrm{mol}$ of BC-fluorescein in $39 \%$ yield.

\section{Age-dependent labeling of SNAP- and CLIP-tagged proteins}

Both Insulin-SNAP and phogrin-CLIP were labeled as previously described ${ }^{5,6,7}$. Briefly, INS-1 medium was changed for medium containing either non-fluorescent CLIP/SNAP-Cell Block (1:400), SNAP-Cell 430 (1:500), CLIP-Cell TMR-Star (1:1000) or synthesized CLIPFluorescein (1:1000) as indicated in the text. After 30 min the medium was aspirated, the cells washed twice with PBS and replaced by non-modified medium. This procedure was repeated twice for $30 \mathrm{~min}$ and once for $60 \mathrm{~min}$.

\section{Sample preparation for fluorescence microscopy, image acquisition and analysis}

For SIM imaging, phogrin-CLIP cells were grown on high precision coverslips coated with polyornithine until a confluency of about $70-80 \%$. The cells were then labeled with the respective SNAP/CLIP substrates as indicated above. The cells were fixed with 4\% PFA for 20 min and permeabilized with $0.1 \%$ Triton X-100 for $10 \mathrm{~min}$. The permeabilized cells were blocked for 1 hour with $0.2 \%$ (fish skin) gelatine solution $+0.5 \%$ BSA in PBS at RT. The primary antibodies were diluted in blocking solution and the cells labeled for $30 \mathrm{~min}$ at RT. Secondary antibody staining was performed similarly for 20 min. For staining of EEA1 (Thermo Scientific, MA5- 
14794) and LAMP2 (Thermo Scientific, PA1-655) permeabilization was ensured by the presence of $0.1 \%$ saponin. Anti-insulin was from Sigma (I-2018) and anti-SNAP from NEB (P9310S). The coverslips were mounted on glass slides with VectaShield Antifade Mounting Medium and fixed with nail polish. Images were acquired using a DeltaVision OMX SIM with an Olympus Plan ApochromatN 60x oil objective with a NA of 1.42. Stacks with a z step-size of $125 \mathrm{~nm}$ were acquired and reconstructed with the SoftWoRx software package (SoftWoRx, Germiston, South Africa). Images were further processed and analyzed using FIJI and R. Contrast was changed for representation purposes. Quantification was performed on background-substracted raw images.

\section{Insulin SG purification for SDS-PAGE and Western Blot}

For isolation of insulin SGs, phogrin-CLIP cells were grown in $175 \mathrm{~cm}^{2}$ flasks (typically $3-4$ per condition). After labeling as indicated above, the cells were harvested in homogenization buffer and homogenized with a glass homogenizer. Aliquots of the postnuclear supernatants (input) were taken to adjust protein contents of different treatments. Anti-substrate antibodies and Protein G Dynabeads (ThermoFisher) were added to the suspension and rotated for 1 hour at $4^{\circ} \mathrm{C}$. The suspension-containing tubes are placed in a magnetic rack and the supernatant transferred in a new tube (flow-through). His- or GST-tagged PreScission protease was added and rotated overnight. The eluate was separated from the beads a second round of LAMP2 immunodepletion (anti-LAMP2, Thermo Scientifc, 51-2200) was done accordingly. Depending on the experiment, either the remaining beads or the eluates were boiled in SDS loading buffer. Antibodies against ICA512 and phogrin were self-raised. AntiSNAP was from NEB (P9310S), anti-ChgA from Abcam (ab45179), anti-PDI from Stressgen (SPA-891), anti-GST from Santa Cruz (sc-138), anti-His from Novagen (70796-3), anti-TGN38 from BD Transduction (610898), anti-PC2 from GeneTex (GTX114625), anti-CPE from Sigma (AB5314), anti-gamma-tubulin from Sigma (T-6557), anti-CANX from BD Transduction (610523), anti-ICA69 from Abcam (ab81500), anti-GM130 from BD Transduction (610822) and anti-Syp1 from Synaptic Systems (101011), anti-EEA1 (Thermo Scientific, MA5-14794), anti-LAMP2 (Thermo Scientific, 51-2200).

\section{Real-time PCR}

Real-time PCR was performed using the GoTaq qPCR Master Mix (Promega) according to manufacturer's instructions. Briefly, cDNA from RT reactions was diluted 1:2 in RNase-free water. Triplicate reactions were set up in Semi-skirted 96-Well PCR Plates $(0.2 \mathrm{ml})$ with optical strip caps (Agilent). The PCR reactions were carried out in an AriaMx Real-time PCR System (Agilent). For absolute quantification, serial dilutions of the target sequence cloned into pCRII 
vectors were used. The results were then normalized by parallel amplification of rat $\beta$-actin mRNA.

\section{Insulin secretion}

Static insulin secretion was measured using a rat ultrasensitive insulin ELISA kit (Alpco) according to manufacturer's recommendations.

\section{Sample preparation for EM}

For electron microscopy, insulin SGs were purified as described above. Instead of acetone precipitation, SGs were either used directly for negative staining or fixed for epon embedding. For negative staining of purified granules $5-10 \mathrm{ml}$ of sample was pipetted to a 300 -mesh copper grid covered with a thin carbon-coated and glow discharged formvar film, and incubated for $10 \mathrm{~min}$ to allow granules to sediment and adhere to the film. Liquid was removed with a piece of filter paper, shortly washed with a drop of water $(2 x)$ and stained with $1 \%$ uranyl acetate (UA) in water for about $20 \mathrm{sec}$. UA was removed slowly with filter paper and the grid was air dried before inspection.

Protein $G$ bead bound granules were fixed with a mixture of formaldehyde (FA, prepared from paraformaldehyde prills) and glutaraldehyde (GA) (2\% FA/2\% GA in $100 \mathrm{mM}$ phosphate buffer), centrifuged, and resuspended in lukewarm agarose (2\%). The agarose was cooled down and cut into small blocks for further processing. Samples were postfixed in $2 \%$ aqueous $\mathrm{OsO}_{4}$ solution containing $1.5 \%$ potassium ferrocyanide and $2 \mathrm{mM} \mathrm{CaCl}$. After washes in water, samples were incubated in $1 \%$ thiocarbohydrazide, washed again and contrasted in $2 \%$ osmium in water for a second time ${ }^{31}$. Samples were washed in water, en bloc contrasted with $1 \%$ uranyl acetate/water, washed again in water, dehydrated in a graded series of ethanol, infiltrated in the epon substitute EMbed $812(1+2,1+1,2+1$ epon/ethanol mixtures, $2 x$ pure epon), and finally embedded in flat embedding molds. Samples were cured at $65^{\circ} \mathrm{C}$ in the oven overnight, and ultrathin sections were cut with a Leica UC6 ultramicrotome and collected on formvar-coated slot grids. Sections were contrasted with uranyl acetate and with lead citrate ${ }^{32}$. All samples were imaged either with a FEI Morgagni 268D (Thermo Fisher Scientific, equipped with a Megaview III camera, SIS-Olympus) or with a Jeol JEM1400 Plus (equipped with a Ruby camera, JEOL) both running at $80 \mathrm{kV}$ acceleration voltage. 


\section{Acknowledgments}

We thank Bert Nitzsche for advice for SIM imaging and Maria Heier for technical contributions developing the protocol, Andreas Müller for initial electron microscopy, Carolin Wegbrod and Anke Sönmez for cell culture work. We are further grateful to Katja Pfriem for administrative assistance. This work was supported by the German Center for Diabetes Research (DZD e.V.), which is financed by the German Ministry for Education and Research. Thomas Kurth and the EMF are supported by EFRE (European Fund for Regional Development).

M.N. was the recipient of a predoctoral fellowship from the Dresden International Graduate School for Biomedicine and Bioengineering (DIGS-BB) in the context of the Excellence Initiative of the German Research Foundation (DFG).

\section{Author contributions}

Conceptualization, M.N., M.S; Methodology, M.N.; Validation, M.N.; Formal analysis, M.N.; Investigation, M.N., K.G., J.V. and T.K.; Resources, J.B. and K.J.; Writing - Original Draft, M.N.; Writing - Reviewing \& Editing, M.N. and M.S.; Visualization, M.N.; Supervision, M.S.; Funding Acquisition, M.N. and M.S.

\section{Competing interest statement}

The authors declare no competing interests. 
Fig. 1 Generation and subcellular localization of phogrin-CLIP (A) Representative SIM image of an INS-1 cell stably expressing phogrin-CLIP labeled with CLIP-TMR and co-stained for insulin. (B) Quantification of co-localization of phogrin-CLIP labeled with TMR and various markers. Anti-CLIP antibody served as positive control. Co-localization was calculated using the Manders split coefficient (MSC). (C) Scheme illustrating the strategy used to purify insulin SGs by their age. The cytoplasmic N-terminus of phogrin was tagged with CLIP. Incorporation of a specific cleavage site allows for the elution of intact SGs from the magnetic beads. Immunopurification is performed with a specific antibody against the substrates (e.g. TMR). Data are represented as boxplots with median \pm SD. Statistical analysis corresponds to t-test with $p$-values equaling ${ }^{* * *}<0.001, N S$. $=$ not significant. Cells from three independent experiments $(n=24-32)$ were analyzed. Scale bar $=10 \mu \mathrm{m}$.

Fig. 2 Characterization of enrichment purity. (A) Silver staining of SDS-PAGE with samples from cells labeled either with or without CLIP-Cell TMR-Star. Input (10 $\mu \mathrm{g})$, flow-through (10 $\mu \mathrm{g}$ ) and Protein $\mathrm{G}$ bead fractions were loaded for comparison. The added protease is detected at $\sim 25 \mathrm{kDa}$. (B) Material from SG purification was loaded and probed for phogrin by Western Blot. (C) SGs were purified and analysed by Western Blot for the organelle markers EEA1, TGN38 and CPH or (D) ChgA and PDI. The His- or GST-tagged PreScission protease (PrecHis or -GST) in the eluate served as loading control.

Fig. 3 Ultrastructural analysis of purified insulin SGs. SGs were purified as described and analyzed by electron microscopy. (A/B) Micrographs of ultrathin sections of bead samples that were embedded in epoxy resin prior to the addition of the PreScission protease embedded in epon. (C/D) Negative staining of eluted material from specific samples as shown in A-B. Scale bar $=1 \mu \mathrm{m}(\mathbf{A} / \mathbf{C}), 200 \mathrm{~nm}(\mathbf{B} / \mathbf{D})$.

\section{Supplementary figures}

Fig. S1 Subcellular localization of phogrin-CLIP. Representative SIM images of INS-1 cells stably expressing phogrin-CLIP labeled with CLIP-TMR and co-stained for CLIP tag (A), EEA1 (B) and LAMP2 (C). Scale bar $=10 \mu \mathrm{m}$.

Fig. S2 Biochemical characterization of the stably expressing phogrin-CLIP line. (A) Cell lysates of the phogrin-CLIP clone were either left untreated (-), treated with endoglycosidase $H(E)$ or peptide:N-glycosidase $F(P)$. Differences in glycosylation were detected by electromobility shift in SDS-PAGE and subsequent probing by Western Blot with antibodies recognizing the CLIP tag. (B) Quantitative PCR of gamma tubulin, endogenous phogrin and overexpressed phogrin-CLIP mRNA levels, normalized to beta actin mRNA. (C) Cell lysates 
of non-transfected INS-1 cells and the clone were probed for phogrin-CLIP (left) or endogenous phogrin (right). (D) Cell lysates of INS-1 cells and the clone were probed for SG markers PC2, CPE and ChgA. Tubulin serves as a loading control. (E) Glucose-stimulated insulin secretion was compared between INS-1 cells and the phogrin-CLIP line. The stimulation index and glucose-stimulated total insulin (GSTI) are shown. For quantitative PCR and static insulin secretion, samples from 5 independent experiments were analyzed. Statistical significance was calculated using the Mann-Whitney-Wilcoxon test with p-values equaling ${ }^{*}<0.05,{ }^{* *}<0.01, \mathrm{NS} .=$ not significant.

Fig. S3 Comparison of aging kinetics of phogrin-CLIP and Ins-SNAP. The stably expressing phogrin-CLIP line was transfected with Ins-SNAP and labeled with SNAP-Cell ${ }^{\circledR} 430$ and BCTMR. (A) Representative SIM image when both tags were labeled for an age of 2-4 h or (B) 2-4 $\mathrm{h}$ for phogrin-CLIP and 20-22 $\mathrm{h}$ for Ins-SNAP. (C) Quantification of co-localization as calculated by Manders split coefficient (MSC). Data are represented as boxplots with median \pm SD. Statistical analysis corresponds to t-test with $p$-values equaling ${ }^{* * *}<0.001, N S$. $=$ not significant. Cells from three independent experiments $(n=21-23)$ were analyzed. Scale bar $=$ $10 \mu \mathrm{m}$.

Fig. S4 Characterization of enrichment purity. SGs were purified and probed for different organelle markers by Western Blot. Eluates of samples labeled with, or without TMR were probed for SG markers ChgA (A), ICA69 (A), ICA512 (C) and CPE (B). Eluates were further probed for the Golgi marker GM130 (A) and the ER marker calnexin (CANX) (C). His-tagged PreScission protease (Prec-His) serves as loading control.

Fig. S5 Characterization of enrichment purity. (A) Purified SG lysates were probed for LAMP2, PC2 and Syp1 by Western Blot. (B) SG purification with a subsequent second immunodepletion step with antibodies either against LAMP2 alone or in combination with Syp1. 


\section{Figure 1}

A

A site-specific

protease

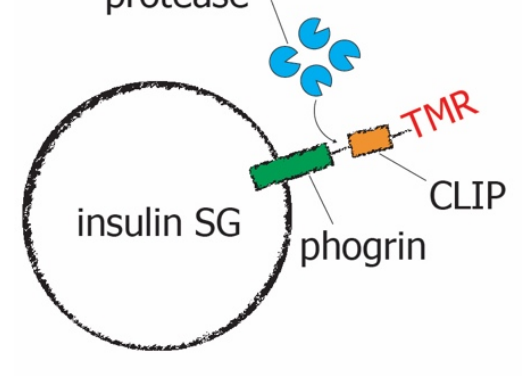

C

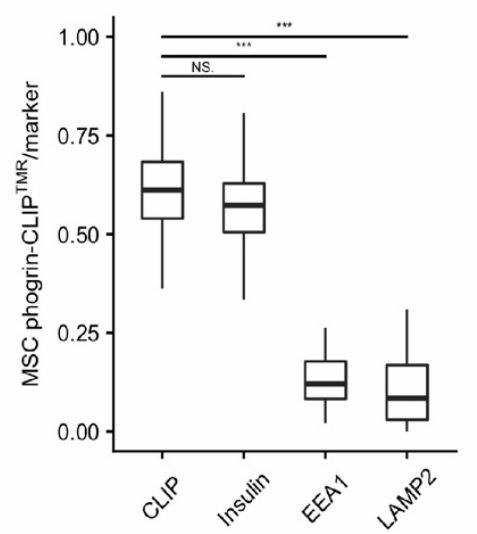

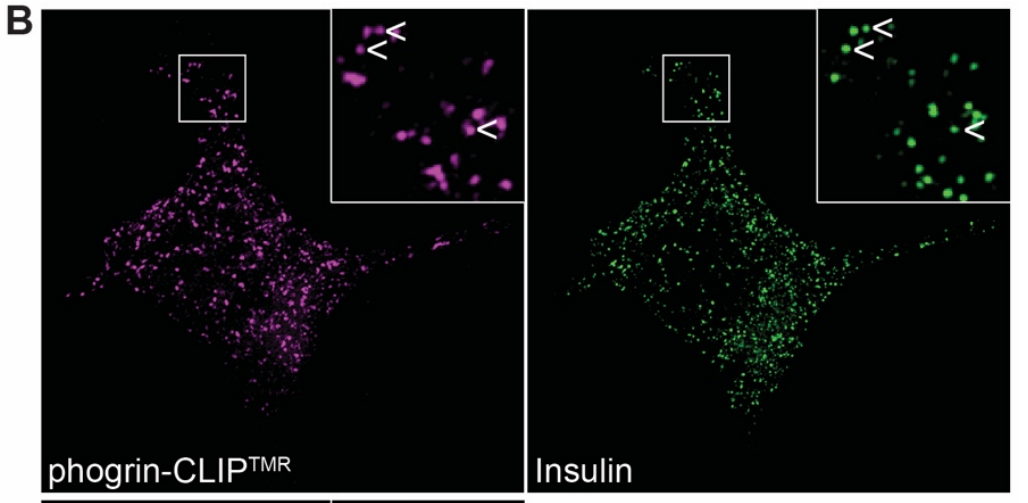

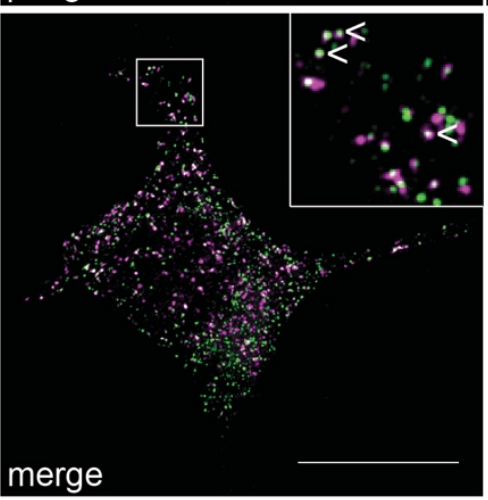




\section{Figure 2}
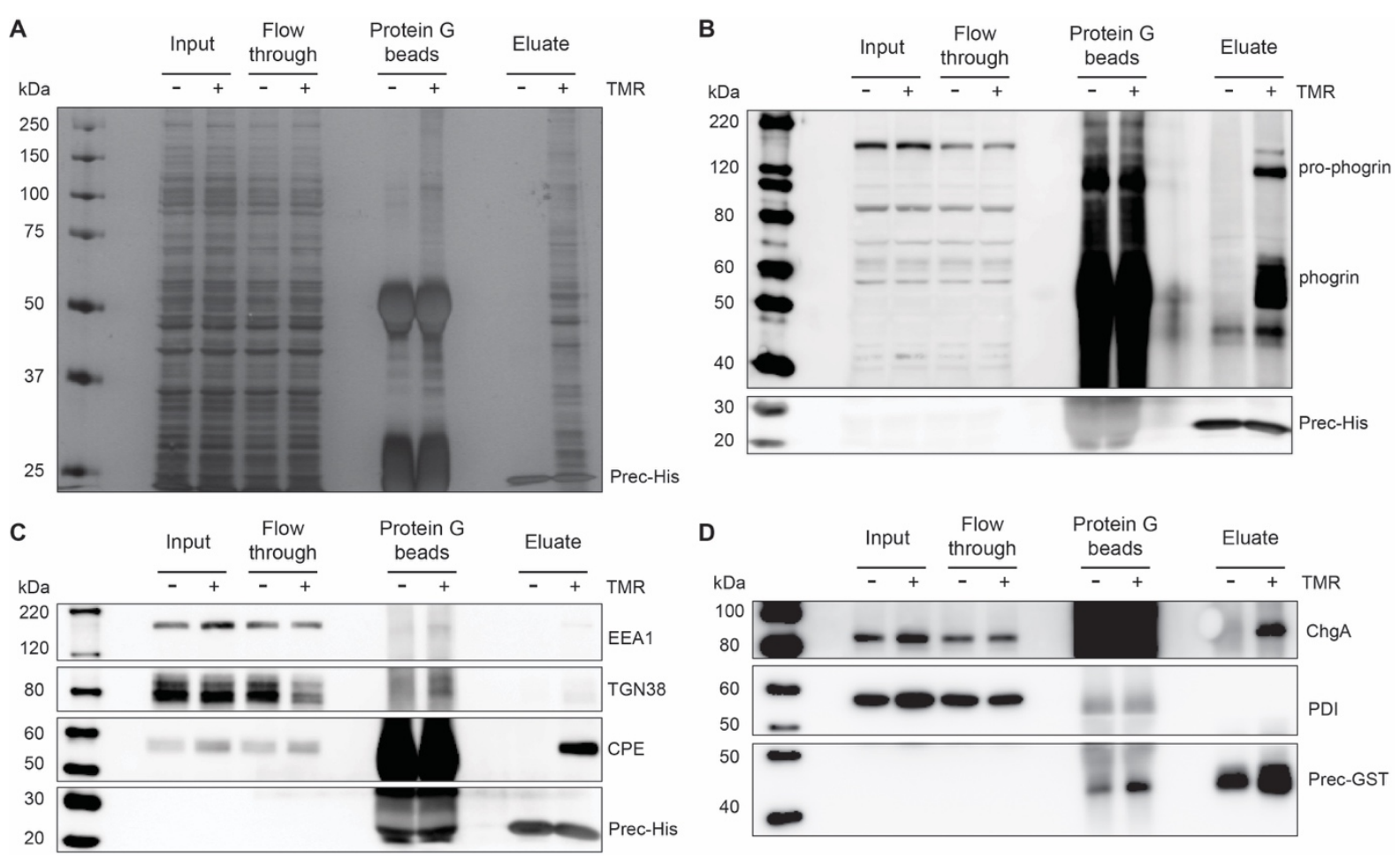


\section{Figure 3}
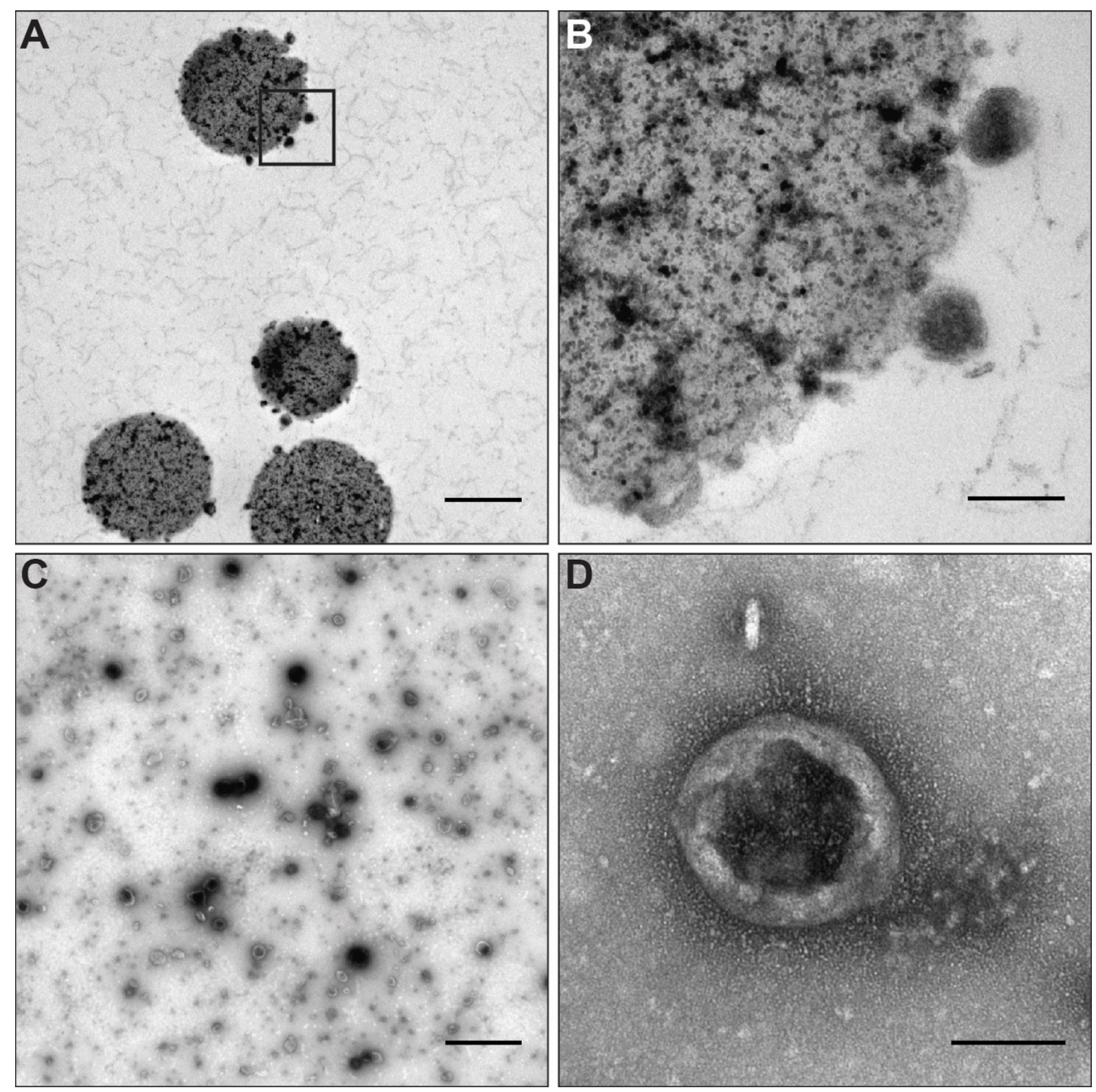


\section{Figure S1}

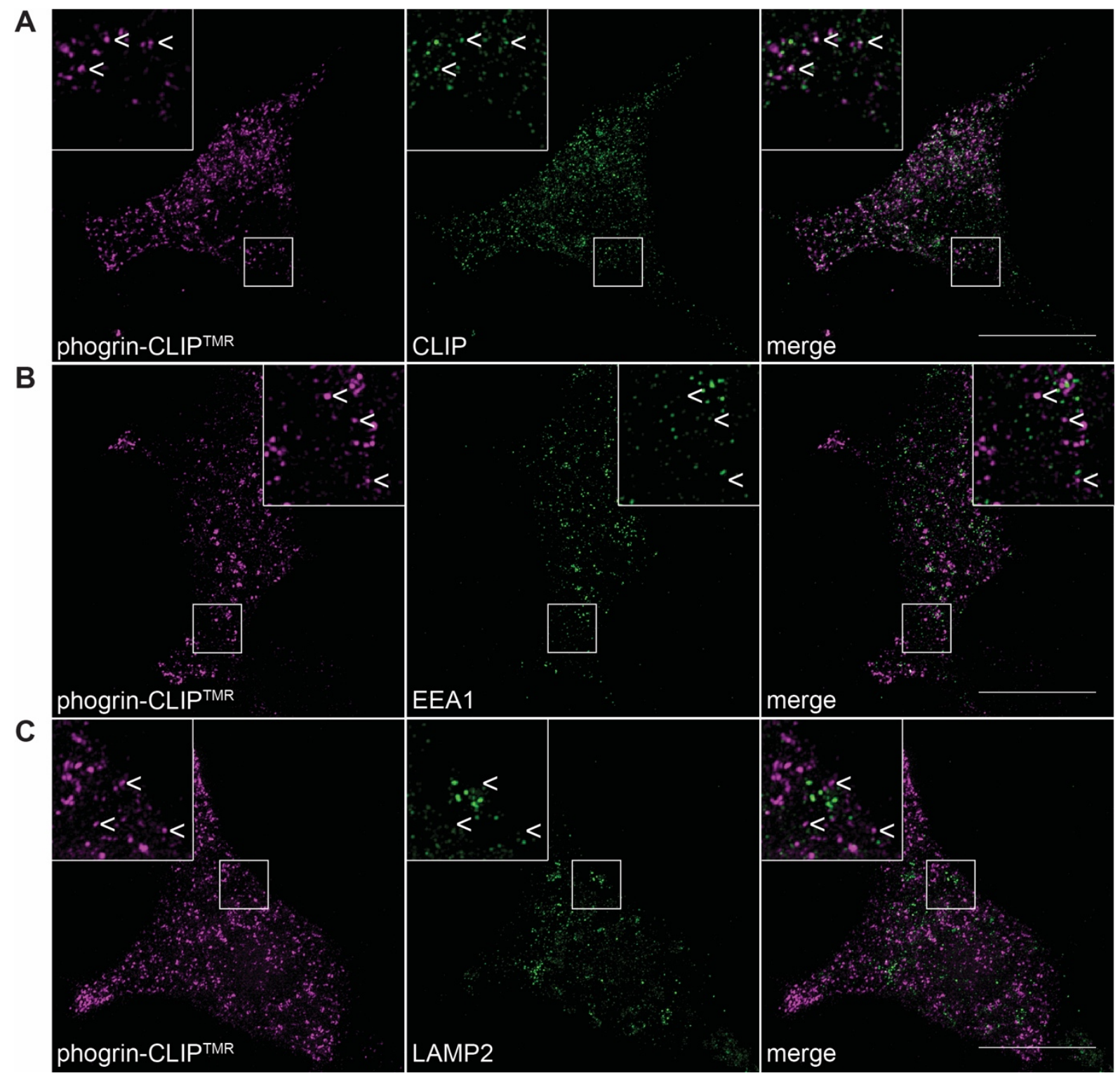




\section{Figure S2}
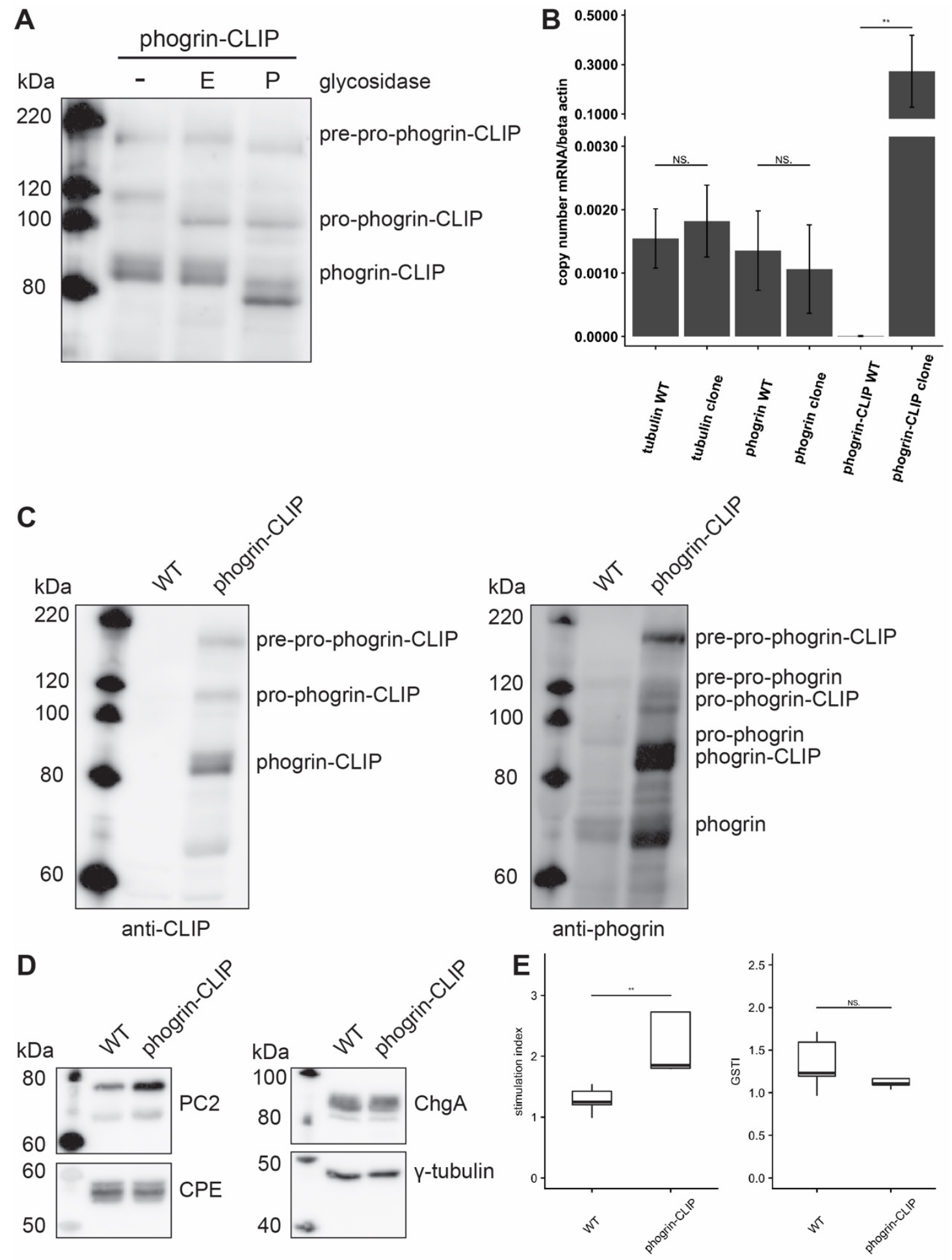


\section{Figure S3}

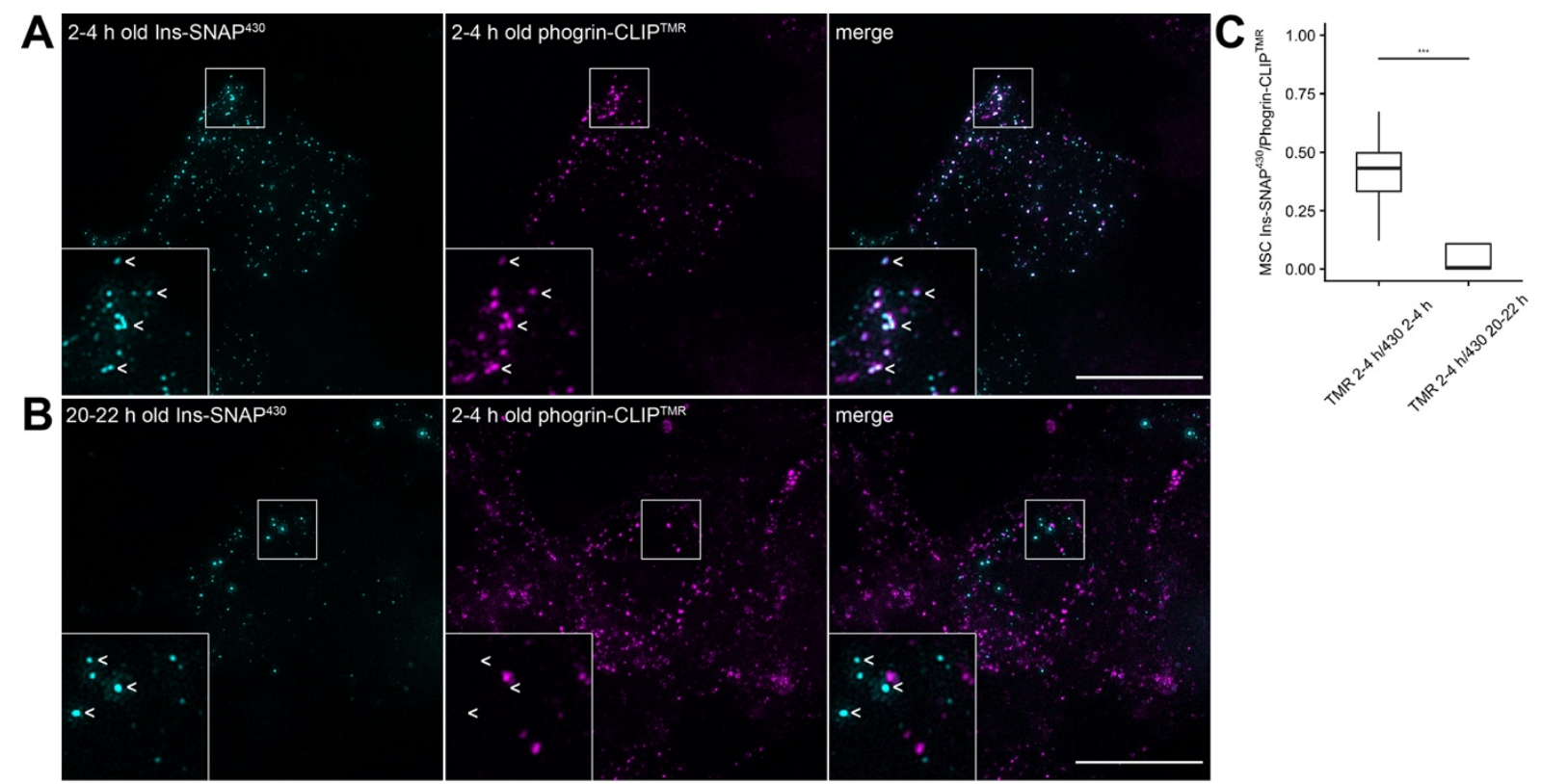

\section{Figure S4}
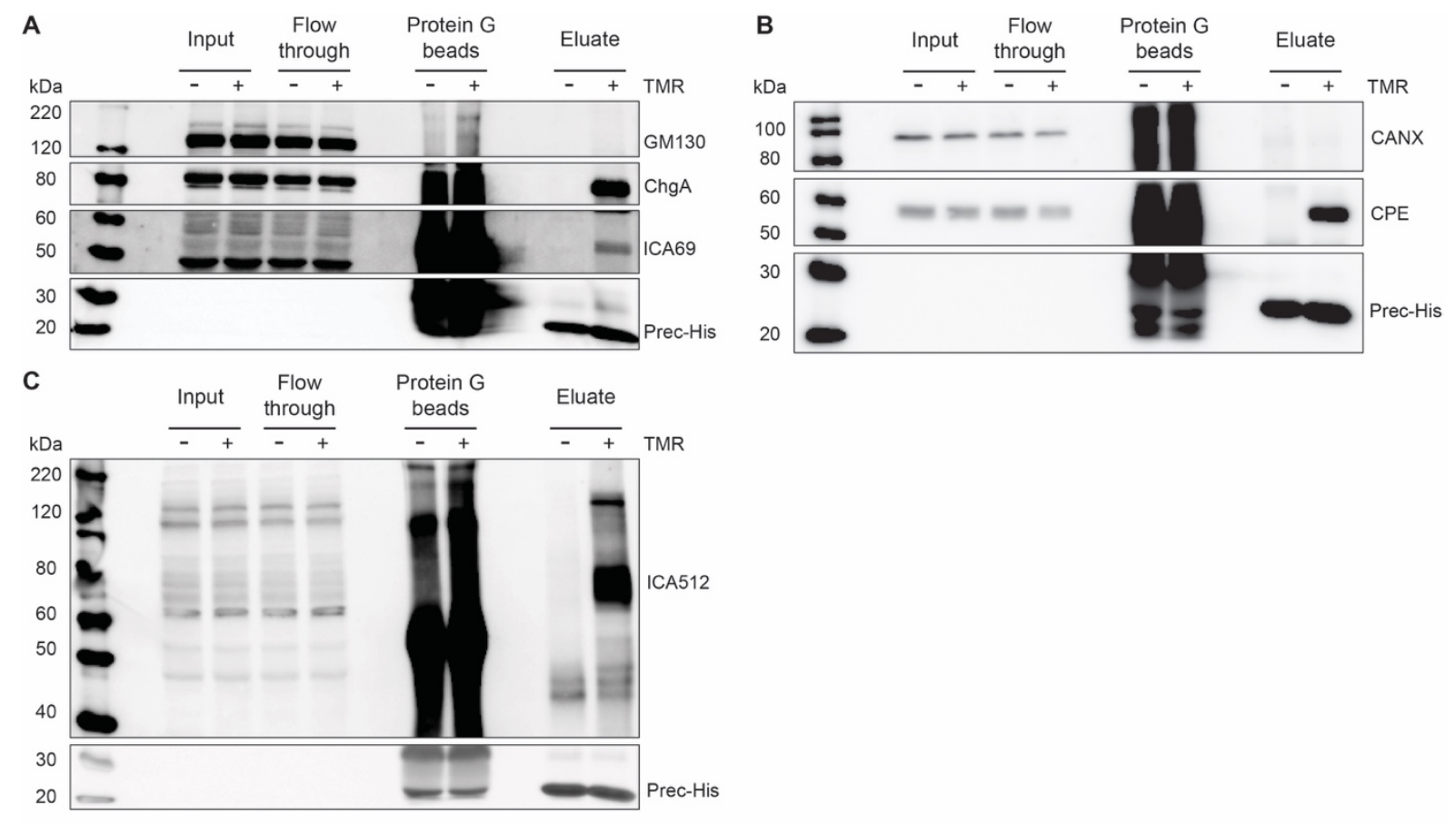
bioRxiv preprint doi: https://doi.org/10.1101/2020.06.03.103770; this version posted June 3, 2020. The copyright holder for this preprint (which was not certified by peer review) is the author/funder. All rights reserved. No reuse allowed without permission.

Figure S5
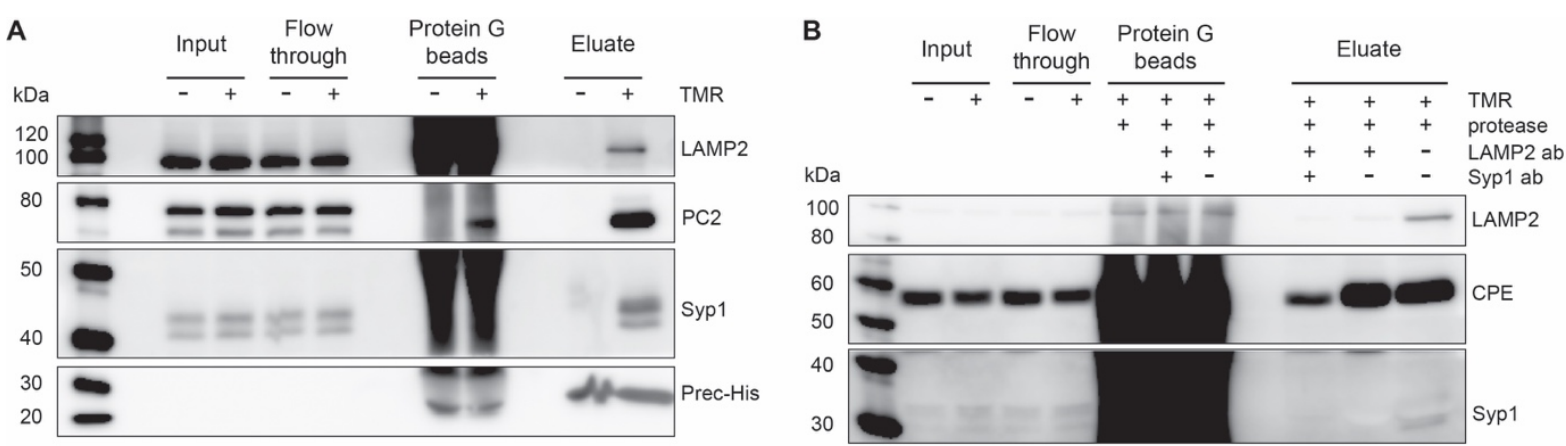